\title{
Quinpi: Integrating Conservation Laws with CWENO Implicit Methods
}

\author{
G. Puppo ${ }^{1}$ (D) M. Semplice ${ }^{2} \cdot$ G. Visconti $^{1}$
}

Received: 31 January 2021 / Revised: 2 August 2021 / Accepted: 4 September 2021 /

Published online: 9 February 2022

(C) The Author(s) 2022

\begin{abstract}
Many interesting applications of hyperbolic systems of equations are stiff, and require the time step to satisfy restrictive stability conditions. One way to avoid small time steps is to use implicit time integration. Implicit integration is quite straightforward for first-order schemes. High order schemes instead also need to control spurious oscillations, which requires limiting in space and time also in the linear case. We propose a framework to simplify considerably the application of high order non-oscillatory schemes through the introduction of a low order implicit predictor, which is used both to set up the nonlinear weights of a standard high order space reconstruction, and to achieve limiting in time. In this preliminary work, we concentrate on the case of a third-order scheme, based on diagonally implicit Runge Kutta (DIRK) integration in time and central weighted essentially non-oscillatory (CWENO) reconstruction in space. The numerical tests involve linear and nonlinear scalar conservation laws.
\end{abstract}

Keywords Implicit schemes · Essentially non-oscillatory schemes · Finite volumes · WENO and CWENO reconstructions

Mathematics Subject Classification 65M08 $\cdot 65 \mathrm{M} 20 \cdot$ 35L65 $\cdot 65 \mathrm{~L} 04$

\section{Introduction}

Hyperbolic systems of conservation laws in one dimension can be written in the form

G. Puppo

gabriella.puppo@uniroma1.it

M. Semplice

matteo.semplice@uninsubria.it

G. Visconti

giuseppe.visconti@uniroma1.it

1 Dipartimento di Matematica, Università La Sapienza, P.le Aldo Moro, 5, 00185 Roma, Italy

2 Dipartimento di Scienza e Alta Tecnologia, Università dell'Insubria, Via Valleggio, 11, 22100 Como, Italy 


$$
u_{t}+f(u)_{x}=0
$$

where $u(x, t)$ is the unknown solution, and $f(u)$ is the flux function. The system is hyperbolic provided the Jacobian of $f, J(f)$, has real eigenvalues and a complete set of eigenvectors.

These systems model propagation phenomena, where initial and boundary data travel and interact along the eigenvectors of the system, with finite speed. The eigenvalues of $J(f)$, which depend on the solution itself when the flux is a nonlinear function of $u$, are the propagation speeds of the system. The size of the eigenvalues can span different orders of magnitude in many applications, and this fact introduces difficulties in the numerical integration of hyperbolic systems of PDE's.

In this paper, we will consider the method of lines (MOL), which is a very popular approach in the integration of hyperbolic systems. See for instance the textbook [32], and the classic review [39], but other approaches are also effective, as in [18]. In our case, we introduce a grid in the computational domain of points $x_{j}$. Here for simplicity we will consider a uniform grid, so all points are separated by a distance $h=x_{j}-x_{j-1}$. The computational domain $\Omega$ is thus covered with a mesh of cells $\Omega_{j}=\left[x_{j}-1 / 2 h, x_{j}+1 / 2 h\right]$, such that $\cup_{j} \Omega_{j}=\Omega$. Introducing the cell averages of the exact solution $\bar{u}(t)_{j}=\frac{1}{h} \int_{\Omega_{j}} u(x, t) \mathrm{d} x$, the hyperbolic system (1) can be written as

$$
\frac{\mathrm{d} \bar{u}_{j}}{\mathrm{~d} t}=-\frac{1}{h}\left[f\left(u\left(x_{j}+\frac{1}{2} h, t\right)\right)-f\left(u\left(x_{j}-\frac{1}{2} h, t\right)\right)\right],
$$

which gives the exact evolution of the cell averages in terms of the difference of the fluxes at the cells interfaces. To transform this relation in a numerical scheme, one introduces reconstruction algorithms, $\mathcal{R}$, whose task is to estimate the value of the solution at the cell interfaces from cell averages, and numerical fluxes $\mathcal{F}$, which estimate the flux at the cell interfaces. Thus, the structure of a numerical scheme in the method of lines approach can be written as follows.

i) Define a reconstruction algorithm $\mathcal{R}(\{\bar{u}\})$ such that the exact solution $u$ at time $t$ is approximated with $u(x, t)=\sum_{j} R_{j}(x ; t) \chi_{j}(x)$, where $\chi_{j}$ is the characteristic function of the interval $\Omega_{j}$, and $R_{j}$ is the restriction of $\mathcal{R}$ to the interval $\Omega_{j}$. Typically, $R_{j}$ is a polynomial of degree $d_{j}$, which changes in time because it is defined starting from the timedependent cell averages. In this paper, we will assume that $d_{j} \equiv d$ is constant.

ii) Compute the boundary extrapolated data (BED) at the cell interfaces $u_{j+1 / 2}^{+}=R_{j+1}\left(x_{j}+\frac{h}{2}\right)$ and $u_{j+1 / 2}^{-}=R_{j}\left(x_{j}+\frac{h}{2}\right)$. Recall that the two BED's computed at the same interface are different, with $u_{j+1 / 2}^{+}-u_{j+1 / 2}^{-}=\mathcal{O}\left(h^{d+1}\right)$ when the flow is smooth enough.

iii) Choose a smooth enough numerical flux function $\mathcal{F}(a, b)$ such that $\mathcal{F}(a, a)=f(a)$, with the stability property that $\mathcal{F}$ be an increasing function of the first argument and a decreasing function of the second argument.

iv) Then the solution of the PDE is approximated by the solution of the system of ODE's

$$
\frac{\mathrm{d} \bar{u}_{j}}{\mathrm{~d} t}=-\frac{1}{h}\left(\mathcal{F}_{j+1 / 2}-\mathcal{F}_{j-1 / 2}\right)
$$

with $\mathcal{F}_{j+1 / 2}=\mathcal{F}\left(u_{j+1 / 2}^{-}, u_{j+1 / 2}^{+}\right)$.

It is well known that for explicit schemes the time step $\Delta t$ must satisfy the CFL condition, namely $\lambda=\Delta t / h \leqslant c / \max _{u}\left|f^{\prime}(u)\right|$, for a constant $c, 1 \geqslant c>0$, the Courant number. Thus the numerical speed $1 / \lambda$ must be faster than all the waves present in the 
system. Since the error in the numerical solution depends on the difference between the numerical and the actual speed, it follows that in explicit schemes the waves which are better approximated are the fastest waves.

Several systems of hyperbolic conservation laws are characterized by waves with very different speeds, and in many applications the phenomenon of interest travels with slow speeds, while the fastest waves which impose the CFL condition do not need to be accurately represented. Low Mach problems in gas dynamics and kinetic problems close to equilibrium provide good examples.

Low Mach problems arise in gas dynamics when the flow is close to incompressibility. In these cases the actual speed of the gas is much slower than the acoustic waves, and if one is interested in the movement of the gas, accuracy in the propagation of sound is irrelevant. A huge literature has developed for these problems, here we mention just a few pioneering works, [13,14] and some more recent developments $[1,7,15,16,41]$.

A second example in which fast velocities constrain the CFL condition, but the signals carried by them do not need to be accurately represented, occurs in kinetic problems, especially close to equilibrium. In kinetic problems the typical evolution equation has the form

$$
f_{t}+v \cdot \nabla_{x} f=Q(f, f),
$$

where $f=f(x, t, v)$ is the distribution function for particles located at $x$, with velocity $v$ at time $t$, and $Q(f, f)$ is the collision term, accounting for the interaction between particles during which the microscopic speeds $v$ are modified. When the flow is close to equilibrium, the relevant phenomena travel with macroscopic speeds which have magnitude of order $\int v f(x, t, v) \mathrm{d} v$ and $\left[\int v^{2} f(x, t, v) \mathrm{d} v\right]^{1 / 2}$. Still, the CFL condition is based on the fastest microscopic speeds, which are typically much larger. Several attempts have been proposed to go around this restriction, see the review [17], and [33]. Note that the microscopic speeds appear in the convective term only, and convection is linear in kinetic problems. Thus it is feasible to integrate these equations with implicit schemes, taking advantage of the linearity of the convective terms, as in [36]. As we will see, Quinpi schemes are linear on linear problems. We expect, therefore, that their application will be particularly effective on kinetic problems.

The structure of the convective term of kinetic problems is exploited also by semiLagrangian schemes, as in $[6,25]$, and the papers of the same series. Semi-Lagrangian schemes track characteristics, and therefore they are explicit, while satisfying automatically stability conditions. However, they need to interpolate the solution on the grid, because the foot of the characteristic, from which the solution is carried up to the next time level, typically does not coincide with a grid point. Enforcing a non-oscillatory interpolation will then widen the stencil. Moreover, especially when the problem is stiff, characteristics may cross the boundary of the domain, before hitting the surface of known data. This makes the construction of semi-Lagrangian schemes for problems on bounded domains quite tricky. On other applications of semi-Lagrangian schemes, see also [8].

Another setting in which convection is linear, and therefore more amenable to implicit integration, arises with relaxation systems of the form proposed in [29]. Relaxation leads to systems of PDE's which have a kinetic form with linear transport, as in (4). Besides kinetic problems, diffusive relaxation leads to fast relaxation microscopic speeds, which again prompts the need for implicit time integration.

In this paper, we will propose numerical schemes for the implicit integration in time of hyperbolic systems of equations. This is a preliminary work. For the time being, we will focus on third-order implicit schemes for scalar conservation laws. As noted in [3], 
there are three levels of nonlinearity in high order implicit methods for conservation laws, which make implicit integration particularly challenging. The first level consists in the nonlinearity of the flux function, which is due to the physical structure of the model, and therefore is unavoidable. The other sources of nonlinearity are due to the need to prevent spurious oscillations, arising with high order numerical schemes. Even in the explicit case, non-oscillatory high order schemes must use nonlinear reconstructions. As is well known, nonlinear reconstructions in space are needed even in implicit schemes. Moreover, high order time integrators are typically based on a polynomial approximation of the time derivative. Thus, a nonlinear limiting is needed also in the time reconstruction of the derivative. These problems have been addressed by several authors. We mention work on second-order schemes in [20, 21], where limiters are applied in space and time simultaneously and TVD estimates are derived. An interesting discussion on TVD bounds in space and time can be found in [22], and in the classic paper [27]. See also [24]. A fully nonlinear third-order implicit scheme, which is limited in both space and time simultaneously can be found in [3].

The next section, Sect. 2, contains a discussion of revisited TVD bounds which are at the basis of our approach, and a summary of the choices leading to the final scheme. Section 3 is the main part of the work, and it offers a complete discussion of the bricks composing the proposed Quinpi algorithm. Next, Sect. 4 documents the properties of the scheme with a selection of numerical tests for scalar equations. We end with conclusions and a plan for future work in Sect. 5.

\section{Motivation}

To study TVD conditions for implicit schemes, we consider the linear advection equation $u_{t}+a u_{x}=0$ with $a>0$ and the upwind scheme. In this section, $u_{j}^{n}$ is the numerical approximation to the exact solution, at one of the space-time grid points, namely, $u_{j}^{n} \simeq u\left(x_{j}, t^{n}\right)$, with $x_{j} \in \Omega$, is a grid point in the space mesh, and $t^{n}=n \Delta t, \Delta t$ being the time step. The mesh ratio is $\lambda=\Delta t / h$.

In the explicit case, we have

$$
u_{j}^{n+1}=u_{j}^{n}-\lambda a\left(u_{j}^{n}-u_{j-1}^{n}\right)
$$

and the total variation of $u^{n+1}$ is

$$
\begin{aligned}
\operatorname{TV}\left(u^{n+1}\right) & =\sum_{j}\left|(1-\lambda a)\left(u_{j}^{n}-u_{j-1}^{n}\right)+\lambda a\left(u_{j-1}^{n}-u_{j-2}^{n}\right)\right| \\
& \leqslant|1-\lambda a| \sum_{j}\left|u_{j}^{n}-u_{j-1}^{n}\right|+|\lambda a| \sum_{j}\left|u_{j-1}^{n}-u_{j-2}^{n}\right| .
\end{aligned}
$$

Then, using the fact that $a>0$, periodic boundary conditions, or compact support of the solution, and the CFL condition, one has that TV $\left(u^{n+1}\right) \leqslant \mathrm{TV}\left(u^{n}\right)$.

For the implicit upwind scheme instead, we start from

$$
u_{j}^{n}=u_{j}^{n+1}+\lambda a\left(u_{j}^{n+1}-u_{j-1}^{n+1}\right) .
$$

Now 


$$
\begin{aligned}
\operatorname{TV}\left(u^{n}\right) & =\sum_{j}\left|(1+\lambda a)\left(u_{j}^{n+1}-u_{j-1}^{n+1}\right)-\lambda a\left(u_{j-1}^{n+1}-u_{j-2}^{n+1}\right)\right| \\
& \geqslant|1+\lambda a| \sum_{j}\left|u_{j}^{n+1}-u_{j-1}^{n+1}\right|-|\lambda a| \sum_{j}\left|u_{j-1}^{n+1}-u_{j-2}^{n+1}\right| .
\end{aligned}
$$

Since $a>0$, and applying again periodic boundary conditions, we find TV $\left(u^{n+1}\right) \leqslant \operatorname{TV}\left(u^{n}\right), \forall \lambda$. Thus, the implicit upwind scheme is not only unconditionally stable, but it is also unconditionally total variation non-increasing.

We now consider the explicit Euler scheme with second-order space differencing, using a piecewise limited reconstruction. The numerical solution now is

$$
u_{j}^{n+1}=u_{j}^{n}-\lambda a\left(1-\frac{1}{2} \Phi\left(\theta_{j-1}\right)\right)\left(u_{j}^{n}-u_{j-1}^{n}\right)-\frac{1}{2} \lambda a \Phi\left(\theta_{j}\right)\left(u_{j+1}^{n}-u_{j}^{n}\right),
$$

where $\Phi(\theta)$ is the limiter, and the quantity $\theta_{j}=\frac{u_{j}-u_{j-1}}{u_{j+1}-u_{j}}$, so that the limited slope is $\sigma_{j}=\Phi\left(\theta_{j}\right)\left(u_{j+1}-u_{j}\right)$.

To prove under what conditions the scheme is TVD, following Harten [26], and Sweby [40], one rewrites this scheme as

$$
u_{j}^{n+1}=u_{j}^{n}-C_{j-1}\left(u_{j}^{n}-u_{j-1}^{n}\right)
$$

with

$$
C_{j-1}=\lambda a\left[1-\frac{1}{2}\left(\Phi\left(\theta_{j-1}\right)-\frac{\Phi\left(\theta_{j}\right)}{\theta_{j}}\right)\right] .
$$

Computing the total variation of the scheme above, one finds that the scheme is TVD provided

$$
C_{j} \geqslant 0, \quad \text { and } \quad 1-C_{j} \geqslant 0 .
$$

Since $a>0$, the first condition holds provided

$$
\alpha_{j}=1-\frac{1}{2}\left(\Phi\left(\theta_{j-1}\right)-\frac{\Phi\left(\theta_{j}\right)}{\theta_{j}}\right) \geqslant 0 .
$$

From this, one recovers the familiar restrictions in the choice of the limiter function, namely $0 \leqslant \frac{\Phi(\theta)}{\theta} \leqslant 2$ and $0 \leqslant \Phi(\theta) \leqslant 2$, see also LeVeque [32]. With these bounds, one has $0 \leqslant \alpha_{j} \leqslant 2$, and the second condition on $C_{j}$ is satisfied provided a slightly restricted CFL holds, namely, $\lambda \leqslant 1 /(2 a)$.

How do these estimates change in the implicit case? Now we have

$$
u_{j}^{n}=u_{j}^{n+1}+\lambda a\left(1-\frac{1}{2} \Phi\left(\theta_{j-1}\right)\right)\left(u_{j}^{n+1}-u_{j-1}^{n+1}\right)+\frac{1}{2} \lambda a \Phi\left(\theta_{j}\right)\left(u_{j+1}^{n+1}-u_{j}^{n+1}\right),
$$

and introducing the same quantity $C_{j}$ we saw before, this expression can be rewrited as

$$
u_{j}^{n}=u_{j}^{n+1}+C_{j-1}\left(u_{j}^{n+1}-u_{j-1}^{n+1}\right) .
$$

The total variation of this scheme is 


$$
\begin{aligned}
\operatorname{TV}\left(u^{n}\right) & =\sum_{j}\left|\left(1+C_{j-1}\right)\left(u_{j}^{n+1}-u_{j-1}^{n+1}\right)-C_{j-2}\left(u_{j-1}^{n+1}-u_{j-2}^{n+1}\right)\right| \\
& \geqslant \sum_{j}\left|1+C_{j-1}\right|\left|u_{j}^{n+1}-u_{j-1}^{n+1}\right|-\sum_{j}\left|C_{j-2}\right|\left|u_{j-1}^{n+1}-u_{j-2}^{n+1}\right|,
\end{aligned}
$$

where we have used the inverse triangle inequality. Applying periodic boundary conditions, and assuming that

$$
C_{j} \geqslant 0, \quad \text { and } \quad 1+C_{j} \geqslant 0,
$$

one finds that the scheme is TVD. The important point for the development in this work is that the first condition gives exactly the same restrictions on the choice of the limiter function we find in the explicit case. The second condition instead is always satisfied. This means that the implicit scheme with piecewise linear reconstruction in space is TVD for all $\lambda$, provided the limiter function satisfies the usual bounds holding for explicit schemes.

The limiter is subject also to accuracy constraints. We achieve second-order accuracy if the limited slope is a first-order approximation of the exact slope. Namely, if $U(x)$ is a smooth function with cell averages $\bar{u}_{j}$, second-order accuracy requires that

$$
U\left(x_{j+1 / 2}\right)-\left(\bar{u}_{j}+\frac{1}{2} \sigma_{j}\right)=\mathcal{O}(h)^{2} .
$$

Substituting the limited slope and expanding around $U\left(x_{j}\right)=u_{j}$, one finds

$$
U\left(x_{j+1 / 2}\right)-u_{j+1 / 2}^{-}=\frac{h}{2}(1-\Phi(1))+\mathcal{O}(h)^{2} .
$$

As in the explicit case, we see that second-order accuracy requires $\Phi(1)=1$, together with at least Lipshitz continuity for $\Phi$. This means that $\Phi$ and $\theta$ can be computed using auxiliary functions that must be at least first order approximations of the unknown function $u\left(x, t^{n+1}\right)$. For these reasons, in this work we will get as follows.

- Build implicit schemes for stiff hyperbolic systems with unconditional stability, based on diagonally implicit Runge Kutta (DIRK) schemes.

- Prevent spurious oscillations in space using exactly the same techniques for space limiting we are familiar with. In fact, as pointed out in [24], Runge Kutta schemes can be seen as combinations of Euler steps. This is true also in the implicit case. We will strive to ensure that each implicit Euler step composing the whole Runge Kutta step prevents spurious oscillations. In other words, we will rewrite the PDE as a system of ODE's in the cell averages, with a non-oscillatory right-hand side.

- The nonlinearities in the space reconstruction will be tackled using first-order predictors, that can be computed without limiting, because, as we have recalled, they are unconditionally TVD. They allow to compute each DIRK stage by solving a system which is nonlinear only because of the nonlinear flux function, and, at the same time, they will constitute a low order non-oscillatory approximation of the solution in its own right.

- As is known, this is not enough to prevent spurious oscillations, when high order space reconstructions are coupled with high order time integrators. A high order Runge Kutta scheme can be viewed as a polynomial reconstruction in time through natural continuous extensions [35, 42]. Not surprisingly, limiting is needed also in time, as pointed out in [20] and more recently [3]. Unlike these authors, however, we limit the solution in 
time applying the limiter directly on the computed solution, blending the accurate solution with the low order predictor, without coupling space and time limiting.

We will name the resulting schemes Quinpi, for central weighted essentially non-oscillatory (CWENO) implicit. In this paper, we will introduce only third-order Quinpi schemes. We plan to extend the construction to include higher-order schemes, BDF extensions and applications in future works.

\section{Quinpi Finite Volume Scheme}

This section is devoted to the description of the implicit numerical solution of a onedimensional scalar conservation law of the form (1) with the Quinpi approach. As pointed out in Sect. 1, we focus on the finite volume framework employing the method of lines, which leads to the system of ODEs (3) for the evolution of the approximate cell averages.

In the following subsections, we will initially describe the space reconstruction for a function $u(x)$, with limiters based on a predicted solution $p(x)$, where $p$ should be at least a first-order approximation of $u$. We will concentrate on the third order CWENO reconstruction [34]. For the general CWENO algorithm, see [10]. More details, improvements and extensions can be found in $[9,11,12,19,31,37]$. Other non-oscillatory reconstructions can also be used, such as WENO, see the classic review [39]. and its extensions as [4].

Next, we will consider the integration in time, with a DIRK3 scheme, describing the interweaving of predictor and time advancement of the solution. Finally, we will introduce the limiting in time, which consists in a nonlinear blending between the predicted and the high order solution, and the conservative correction that is needed at the end of the time step.

\subsection{Space Approximation: the CWENO Reconstruction Procedure}

The CWENO schemes are a class of high-order numerical methods to reconstruct accurate and non-oscillatory point values of a function $u$ starting from the knowledge of its cell averages. The main characteristic of CWENO reconstructions is that they are uniformly accurate within the entire cell, [10].

For the purpose of this work, here we briefly recall the definition of the CWENO reconstruction procedure restricting the presentation to the one-dimensional third-order scalar case on a uniform mesh. In order to reconstruct a function $u$ at some $x \in \Omega$ and at a fixed time $t$, we consider as given data the cell averages $\bar{u}_{j}$ of $u$ at time $t$ over the cells $\Omega_{j}$ of a grid, which is a uniform discretization of $\Omega$.

A third-order CWENO reconstruction is characterized by the use of an optimal polynomial of degree 2 and two linear polynomials. Let $P_{j, L}^{(1)}$ and $P_{j, R}^{(1)}$ be the linear polynomials

$$
\left\{\begin{array}{c}
P_{j, L}^{(1)}=\bar{u}_{j}+\frac{\bar{u}_{j}-\bar{u}_{j-1}}{h}\left(x-x_{j}\right), \\
P_{j, R}^{(1)}=\bar{u}_{j}+\frac{\bar{u}_{j+1}-\bar{u}_{j}}{h}\left(x-x_{j}\right),
\end{array}\right.
$$

and let us write the optimal polynomial of degree 2 as 


$$
\left\{\begin{aligned}
P_{j}^{(2)}(x) & =a+b\left(x-x_{j}\right)+c\left(x-x_{j}\right)^{2}, \\
a & =\frac{-\bar{u}_{j+1}+26 \bar{u}_{j}-\bar{u}_{j-1}}{\bar{u}_{j+1}-\bar{u}_{j-1}^{2}}, \\
b & =\frac{\bar{u}_{j+1}-2 h}{2 \bar{u}_{j}+\bar{u}_{j-1}} .
\end{aligned}\right.
$$

All polynomials $P_{j, L}^{(1)}, P_{j, R}^{(1)}$ and $P_{j}^{(2)}$ interpolate the data in the sense of cell averages. We also introduce the second degree polynomial $P_{j, 0}$ defined as

$$
\left\{\begin{aligned}
P_{j, 0}(x) & =\frac{1}{C_{0}}\left(P_{j}^{(2)}(x)-C_{L} P_{j, L}^{(1)}-C_{R} P_{j, R}^{(1)}\right)=A+B\left(x-x_{j}\right)+C\left(x-x_{j}^{2}\right), \\
A & =\frac{a}{C_{0}}-\frac{C_{L}+C_{R}}{C_{0}} \bar{u}_{j}, \\
B & =\frac{\left(1-2 C_{R}\right) \bar{u}_{j+1}-\left(2 C_{L}-2 C_{R}\right) \bar{u}_{j}+\left(2 C_{L}-1\right) \bar{u}_{j-1}}{2 C_{0} h}, \\
C & =\frac{c}{C_{0}},
\end{aligned}\right.
$$

where $C_{0}, C_{L}, C_{R} \in(0,1)$ with $C_{0}+C_{L}+C_{R}=1$ are the linear or optimal coefficients. Note that this polynomial reproduces the underlying data with second order accuracy.

The Jiang-Shu smoothness indicators [28] of the polynomials related to the cell $\Omega_{j}$ are defined by

$$
I\left[P^{(r)}\right]:=\sum_{i=1}^{r} h^{2 i-1} \int_{\Omega_{j}}\left(\frac{\mathrm{d}^{i}}{\mathrm{~d} x^{i}} P^{(r)}(x)\right)^{2} \mathrm{~d} x .
$$

Then, in our case, they reduce to

$$
I_{j, L}=\left(\bar{u}_{j}-\bar{u}_{j-1}\right)^{2}, \quad I_{j, R}=\left(\bar{u}_{j+1}-\bar{u}_{j}\right)^{2}, \quad I_{j, 0}=b^{2} h^{2}+\frac{52}{3} c^{2} h^{4} .
$$

From these, the nonlinear weights are defined as

$$
\tilde{\omega}_{j, k}=\frac{C_{k}}{\left(\epsilon_{x}+I_{j, k}\right)^{\tau}}, \quad \omega_{j, k}=\frac{\tilde{\omega}_{j, k}}{\tilde{\omega}_{j, 0}+\tilde{\omega}_{j, L}+\tilde{\omega}_{j, R}}, \quad k=0, L, R .
$$

Following $[11,12,31]$, we will always set $\epsilon_{x}=h^{2}$ and $\tau=2$. The reconstruction polynomial $P_{j, \text { rec }}$ is

$$
P_{j, \mathrm{rec}}(x)=\omega_{j, 0} P_{j, 0}(x)+\omega_{j, L} P_{j, L}^{(1)}(x)+\omega_{j, R} P_{j, R}^{(1)}(x) .
$$

Note that if $\omega_{j, k}=C_{k}, k=0, L, R$, then the reconstructed polynomial $P_{j \text {,rec }}$ coincides with the optimal polynomial $P_{j}^{(2)}$, and the reconstruction is third order accurate in the whole cell.

If the nonlinear weights satisfy

$$
C_{k}-\omega_{j, k}=\mathcal{O}(h),
$$

then CWENO boosts the accuracy of the reconstruction polynomial $P_{\text {rec }}$ to the highest possible accuracy, 3 in this case. This occurs when the stencil containing the data is smooth.

Once the reconstruction is known, we can compute the boundary extrapolated data as

$$
u_{j+1 / 2}^{-}=P_{j, \text { rec }}\left(x_{j+1 / 2}\right), \quad u_{j+1 / 2}^{+}=P_{j+1, \text { rec }}\left(x_{j+1 / 2}\right) .
$$


In Quinpi methods, we suppose we are given a predictor $p(x)$ alongside the solution $u(x)$, where the predictor is at least a first-order approximation of the solution. We compute the smoothness indicators (8) using the predictor. Thus, the weights $\omega_{j, k}$ depend only on the predictor. In this fashion, the boundary extrapolated data (13) are linear functions of the solution $u$ with coefficients that are not constant across the grid.

\subsection{Time Approximation: Diagonally Implicit Runge-Kutta (DIRK)}

The space reconstruction algorithm allows to compute boundary extrapolated data of the solution $u$ of the conservation law (1) at each cell interface. Next we pick a consistent and monotone numerical flux function $\mathcal{F}$. For example, in this work, we will apply the Lax Friedrichs numerical flux

$$
\mathcal{F}\left(u^{-}, u^{+}\right)=\frac{1}{2}\left(f\left(u^{+}\right)+f\left(u^{-}\right)-\alpha\left(u^{+}-u^{-}\right)\right)
$$

with $\alpha=\max _{u}\left|f^{\prime}(u)\right|$.

This completely defines the system of ODE's (3). This system needs to be approximated in time by means of a time integration scheme. Here, we focus on DIRK methods, with $s$ stages and general Butcher tableau

$$
\begin{array}{c|cccc}
c_{1} & a_{11} & 0 & \ldots & 0 \\
c_{2} & a_{21} & a_{22} & \ldots & 0 \\
\vdots & \vdots & \vdots & & \vdots \\
c_{s} & a_{s 1} & a_{s 2} & \ldots & a_{s s} \\
\hline & & & & \\
& b_{1} & b_{2} & \ldots & b_{s}
\end{array}
$$

having the property $a_{k, \ell}=0$, for each $k<\ell$.

Discretization of (3) with a DIRK method leads to the fully discrete scheme

$$
\bar{u}_{j}^{n+1}=\bar{u}_{j}^{n}-\frac{\Delta t}{h} \sum_{k=1}^{s} b_{k}\left[\mathcal{F}_{j+\frac{1}{2}}^{(k)}-\mathcal{F}_{j-\frac{1}{2}}^{(k)}\right], \quad n \geqslant 0, j=1, \cdots, N,
$$

where we recall that $h$ is the mesh spacing, $\Delta t$ is the time step, and $\bar{u}_{j}^{n} \approx \bar{u}_{j}\left(t^{n}\right)$. Finally, $\mathcal{F}_{j+\frac{1}{2}}^{(k)}=\mathcal{F}\left(u_{j+\frac{1}{2}}^{-,(k)}, u_{j-\frac{1}{2}}^{+,(k)}\right)$, where the boundary extrapolated data $u_{j+\frac{1}{2}}^{-,(k)}, u_{j-\frac{1}{2}}^{+,(k)}$, are reconstructions at the cell boundaries of the stage values

$$
\bar{u}_{j}^{(k)}=\bar{u}_{j}^{n}-\frac{\Delta t}{h} \sum_{\ell=1}^{k} a_{k, \ell}\left[\mathcal{F}_{j+\frac{1}{2}}^{(\ell)}-\mathcal{F}_{j-\frac{1}{2}}^{(\ell)}\right], \quad k=1, \cdots, s,
$$

which are approximations at time $t^{(k)}=t^{n}+c_{k} \Delta t$. We point out that $\mathcal{F}_{j+\frac{1}{2}}^{(k)}$ depends on the $k$ th stage value, and thus the computation of each stage is implicit but independent from the following ones.

The DIRK scheme used in this work is 


$$
\begin{array}{c|ccc}
\lambda & \lambda & 0 & 0 \\
\frac{1+\lambda}{2} & \frac{1-\lambda}{2} & \lambda & 0 \\
1 & -\frac{3}{2} \lambda^{2}+4 \lambda-\frac{1}{4} & \frac{3}{2} \lambda^{2}-5 \lambda+\frac{5}{4} & \lambda \\
\hline & -\frac{3}{2} \lambda^{2}+4 \lambda-\frac{1}{4} & \frac{3}{2} \lambda^{2}-5 \lambda+\frac{5}{4} \lambda
\end{array}
$$

with $\lambda=0.4358665215$, see [2].

The fully discrete set of Eqs. (16), (17) has two sources of nonlinearity when solved with high-order schemes: one arises from the physics of the system (1) when the flux function $f$ is nonlinear, and cannot be avoided; the other one arises from the computation of the boundary extrapolated data using a high order CWENO or WENO reconstruction, which is nonlinear even for linear problems. Therefore, even for a linear PDE, the resolution of (16), (17) requires a nonlinear solver. Typically, one uses Newton's algorithm, which requires the computation of the Jacobian of the scheme, which depends on the nonlinear weights (10) and on the oscillation indicators (8), (9), resulting in a prohibitive computational cost.

In the next subsection, we propose a way to circumvent the nonlinearity with a highorder reconstruction procedure relying on a predictor.

\subsection{Third-Order Quinpi Approach}

A prototype of an implicit scheme for WENO reconstructions based on a predictor was developed by Gottlieb et al. [23]. The method relies on the idea of a predictor-corrector approach to avoid the nonlinearity of the reconstruction. The solution of an explicit scheme is used as predictor to compute the nonlinear weights of WENO within the high-order implicit scheme, which is used as corrector.

In [23] the approximation with the explicit predictor is computed in a single time step, namely, without performing several steps within the whole time step. On the contrary, in the Quinpi approach, the nonlinearity arising from the nonlinear weights of CWENO is circumvented by computing an approximation of the solution at each intermediate time $t^{(k)}=t^{n}+c_{k} \Delta t$ with an implicit, but linear, low-order scheme, with which the nonlinear weights of CWENO are predicted at each stage.

Once the weights are known, a correction of order 3 is obtained by employing a DIRK method of order three coupled with the third-order CWENO space reconstruction, with the weights computed from the predictor.

In this way, the complete scheme is linear with respect to the space reconstruction. In this context, by linear we mean that, for a linear conservation law, the solution can be advanced by a time step solving a sequence of $s$ narrow-banded linear systems. However, the scheme overall is nonlinear with respect to its initial data because the entries of the linear systems' matrices depend nonlinearly on the predicted solution through (10) and (9). When $f(u)$ is not linear, the systems become nonlinear but only through the flux function.

Clearly, an implicit predictor is more expensive to compute than the explicit predictor proposed in [23]. But using an implicit predictor has a double advantage. First, the predictor itself is stable, and this allows to have a reliable prediction of the weights, even for high Courant numbers. Second, at the end of the time step, the predictor itself is a reliable, stable and nonoscillatory low order solution, with which we will blend the high order solution to obtain the time limiting required by high order time integrators. 


\subsubsection{First-Order Predictor: Composite Implicit Euler}

We solve (14), (16), (17), within the time step $\Delta t$ using an $s$ stages composite backward Euler scheme in time, where $s$ is the number of stages in the DIRK scheme (15). In other words, we apply the backward Euler scheme $s$ times in each time step. The $k$-th substep advances the solution from $t^{n}+\tilde{c}_{k-1} \Delta t$ to $t^{n}+\tilde{c}_{k} \Delta t$, with $\tilde{c}_{0}=0$, where the coefficients $\tilde{c}_{k}, k=1, \cdots, s$, are the ordered abscissae of the DIRK (15). Overall, this is equivalent to implementing a DIRK scheme with Butcher tableau given by

$$
\begin{array}{c|cccc}
\tilde{c}_{1} & \tilde{c}_{1} & 0 & \ldots & 0 \\
\tilde{c}_{2} & \tilde{c}_{1} & \tilde{c}_{2}-\tilde{c}_{1} & \ldots & 0 \\
\vdots & \vdots & \vdots & & \vdots \\
\tilde{c}_{s} & \tilde{c}_{1} & \tilde{c}_{2}-\tilde{c}_{1} & \ldots & \tilde{c}_{s}-\tilde{c}_{s-1} \\
\hline & & & & \\
& \tilde{c}_{1} & \tilde{c}_{2}-\tilde{c}_{1} & \ldots & \tilde{c}_{s}-\tilde{c}_{s-1}
\end{array}
$$

In space, we use piecewise constant reconstructions from the cell averages. At the final stage, we thus obtain a first-order stable non-oscillatory approximation of the solution at the time step $t^{n+1}$, which we will call $u^{\mathrm{IE}, n+1}$. A similar low order composite backward Euler scheme is also employed in the third-order scheme of Arbogast et al. [3].

The resulting scheme also provides first-order approximations $\bar{u}_{j}^{\mathrm{IE},(k)}$ of the solution at the intermediate time $t^{(k)}=t^{n}+\tilde{c}_{k} \Delta t$, for $k=1, \cdots, s$. At each stage therefore one needs to solve the nonlinear system

$$
\begin{aligned}
G_{j}\left(\bar{U}^{\mathrm{IE},(k)}\right):= & \frac{\theta_{k} \Delta t}{h}\left[\frac{1}{2} f\left(\bar{u}_{j+1}^{\mathrm{IE},(k)}\right)-\frac{1}{2} f\left(\bar{u}_{j-1}^{\mathrm{IE},(k)}\right)-\frac{\alpha}{2} \bar{u}_{j+1}^{\mathrm{IE},(k)}\right. \\
& \left.+\left(\alpha+\frac{h}{\theta_{k} \Delta t}\right) \bar{u}_{j}^{\mathrm{IE},(k)}-\frac{\alpha}{2} \bar{u}_{j+1}^{\mathrm{IE},(k)}\right]-\bar{u}_{j}^{\mathrm{IE},(k-1)}=0,
\end{aligned}
$$

where $\theta_{k}:=\tilde{c}_{k}-\tilde{c}_{k-1}, \bar{U}^{\mathrm{IE},(k)}:=\left\{\bar{u}_{j}^{\mathrm{IE},(k)}\right\}_{j}$ and $\bar{u}_{j}^{\mathrm{IE},(0)}:=\bar{u}_{j}^{n}$. We use Newton's method. Note that the system is nonlinear only through the flux function.

\subsubsection{CWENOThird-Order Correction}

Once the low order predictions $\bar{u}_{j}^{\mathrm{IE},(k)}$ are known at all time $t^{(k)}=t^{n}+\tilde{c}_{k} \Delta t$, we correct the accuracy of the solution by solving (14), (16), (17) using the third-order DIRK (18) in time and the third-order CWENO reconstruction in space, with the weights $\omega_{j}^{(k)}$ at the $k$-th stage computed through the predictor $\bar{u}_{j}^{\mathrm{IE},(k)}$. Thus the boundary extrapolated data will be given by

$$
\left\{\begin{array}{l}
u_{j+\frac{1}{2}}^{-,(k)}=P_{j, \mathrm{rec}}\left(x_{j+\frac{1}{2}}\right)=\sum_{\ell=-1}^{1} W_{j, \ell}^{-} \bar{u}_{j+\ell}^{(k)}, \\
u_{j+\frac{1}{2}}^{+,(k)}=P_{j+1, \text { rec }}\left(x_{j+\frac{1}{2}}\right)=\sum_{\ell=-1}^{1} W_{j, \ell}^{+} \bar{u}_{j+1+\ell}^{(k)},
\end{array}\right.
$$

where the weights $W_{j}^{ \pm}$depend only on $\bar{u}_{j}^{\mathrm{IE},(k)}$ and are constant with respect to $\bar{u}^{k}$. Finally, from (14), (16), (17), at each stage, we solve the system 


$$
\begin{aligned}
G_{j}\left(\bar{U}^{(k)}\right):= & \bar{u}_{j}^{(k)}-\bar{u}_{j}^{n} \\
& +\frac{a_{k, k} \Delta t}{h}\left[\mathcal{F}_{j+1 / 2}^{(k)}-\mathcal{F}_{j-1 / 2}^{(k)}\right]+\frac{\Delta t}{h} \sum_{\ell=1}^{k-1} a_{k, \ell}\left[\mathcal{F}_{j+1 / 2}^{(\ell)}-\mathcal{F}_{j-1 / 2}^{(\ell)}\right]=0 .
\end{aligned}
$$

We observe that the nonlinearity of $G_{j}$ is only due to the flux function, and not to the CWENO reconstruction, which uses the predictor $\bar{u}_{j}^{\mathrm{IE},(k)}$ to compute the nonlinear weights.

In the following, the numerical solution obtained applying the DIRK3 scheme, with the third-order CWENO reconstruction exploiting the $u^{\mathrm{IE}}$ predictor will be called D3P1 scheme.

\subsubsection{Nonlinear Blending in Time}

The solution $u^{\mathrm{D} 3 \mathrm{P} 1}$ obtained with the $\mathrm{D} 3 \mathrm{P} 1$ scheme is third-order accurate, and has control over spurious oscillations, thanks to the limited space reconstruction described above. However, this solution may still exhibit oscillations, because it is not limited in time. We discuss in this section the definition of the time-limited solution.

It is easy to associate a continuous extension (CE) to a Runge-Kutta scheme. For example, when the abscissae $c_{k}, k=1, \cdots, s$, are distinct, following [35] one can construct a polynomial $P(t)$ such that $P\left(t^{n}\right)=u^{n}$ and $P^{\prime}\left(t^{n}+c_{k} \Delta t\right)=K_{k}$, where the $K_{k}$ 's are the RK fluxes of the Runge-Kutta scheme and $u^{n}$ is the solution at time $t^{n}$. The polynomial $P(t)$ is such that $P\left(t^{n}+\Delta t\right)=u^{n+1}$ and it provides a way to interpolate the numerical solution at any point $t^{n}+\gamma \Delta t$ for $\gamma \in(0,1)$. If, for a particular Runge-Kutta scheme, a natural continuous extension in the sense of [42] exists, one could use that, but for this work we do not require the extensions to be natural, since we use them only as a device to assess the smoothness of the solution in the time step.

In our case, the CE of the DIRK 3 scheme we are using defines a polynomial extension of degree 3 in the sense of [35], which will be called $P_{t}^{(3)}$. Instead, we name $P_{t}^{(1)}$ the polynomial extension underlying the composite implicit Euler (19).

We define the limited in time solution $u^{B}(t)$ in a CWENO fashion as

$$
u_{j}^{B}(t)=\frac{\omega_{j}^{H, n}}{C_{H}}\left(P_{t}^{(3)}(t)-C_{L} P_{t}^{(1)}(t)\right)+\omega_{j}^{L, n} P_{t}^{(1)}(t), \quad t \in\left[t^{n}, t^{n+1}\right]
$$

with $\omega_{j}^{H, n}+\omega_{j}^{L, n}=1$. In this and in all subsequent equations, $H$ and $L$ stand for high and low order quantities, respectively. The coefficients $C_{L}$ and $C_{H}$ are such that $C_{L}, C_{H} \in(0,1)$ with $C_{L}+C_{H}=1$. We observe that, by a property of the CE polynomials, at time $t^{n+1}$ we have

$$
u_{j}^{B, n+1}=\frac{\omega_{j}^{H, n}}{C_{H}}\left(u_{j}^{\mathrm{D} 3 \mathrm{P} 1, n+1}-C_{L} u_{j}^{\mathrm{IE}, n+1}\right)+\omega_{j}^{L, n} u_{j}^{\mathrm{IE}, n+1} .
$$

Equation (23) describes a nonlinear blending between the low order solution $u^{\mathrm{IE}}$ and the high order solution $u^{\mathrm{D} 3 \mathrm{P} 1}$ at time $t^{n+1}$. We notice that if $\omega_{j}^{H, n}=C_{H}$, and consequently $\omega_{j}^{L, n}=C_{L}$, then $u_{j}^{B, n+1}=u_{j}^{\mathrm{D} 3 \mathrm{P} 1, n+1}$ and the blending selects the solution of the high order scheme. Instead, if $\omega_{j}^{L, n}=1, u_{j}^{B, n+1}=u_{j}^{\mathrm{IE}, n+1}$ and the blending selects the solution of the low order scheme. 
The weights $\omega^{L}$ and $\omega^{H}$ must be thus designed in order to privilege the high order solution when it is not oscillatory, and the low order solution otherwise. In the following we discuss their definition, which is also carried out as in CWENO and relies on suitable regularity indicators. In fact, we define

$$
\omega_{j}^{\ell, n}=\frac{\tilde{\omega}_{j}^{\ell, n}}{\tilde{\omega}_{j}^{L, n}+\tilde{\omega}_{j}^{H, n}}, \quad \ell=L, H,
$$

where

$$
\tilde{\omega}_{j}^{L, n}=\frac{C_{L}}{\epsilon_{t}^{2}}
$$

is the constant weight associated to the first-order non-oscillatory solution $u^{\mathrm{IE}}$ in the time interval $\left[t^{n}, t^{n+1}\right]$, and

$$
\tilde{\omega}_{j}^{H, n}=\frac{C_{H}}{\left(\epsilon_{t}+I_{j}^{3}\right)^{2}}
$$

is the weight associated to the D3P1 approximation in the $j$-th cell in the time interval $\left[t^{n}, t^{n+1}\right]$. Here, $\epsilon_{t}=\Delta t^{\tau}$, and we always take $\tau=2$ if not otherwise stated. The $I_{j}^{3}$ is a smoothness indicator that measures the regularity of the D3P1 solution. We define $I_{j}^{3}$ as the contribution of two terms

$$
I_{j}^{3}=I_{j}^{t}+I_{j}^{x,-}+I_{j}^{x,+},
$$

where $I_{j}^{t}$ and $I_{j}^{x, \pm}$ are smoothness indicators designed in order to detect discontinuity in time and space, respectively, over the cell $j$. The definition of $I_{j}^{t}$ relies on the CE polynomial $P_{t}^{(3)}$. In fact, at each cell, the CE polynomial changes, and we will have different CE's, and each CE will provide local information on the smoothness of the DIRK advancement in time. We measure the regularity of $P_{t}^{(3)}$ by the Jiang-Shu smoothness indicator, namely

$$
I_{j}^{t}=\sum_{\ell=1}^{3}(\Delta t)^{2 \ell-1} \int_{t^{n}}^{t^{n+1}}\left(\frac{\mathrm{d}^{\ell}}{\mathrm{d} t^{\ell}} P_{t}^{(3)}(t)\right)^{2} \mathrm{~d} t .
$$

Instead, the definition of $I_{j}^{x, \pm}$ draws inspiration from [3], and for the DIRK3 method (18) we have

$$
\begin{aligned}
& I_{j}^{x,+}=\left(\bar{u}_{j+1}^{\mathrm{D} 3 \mathrm{P} 1, n}-\bar{u}_{j}^{\mathrm{D} 3 \mathrm{P} 1, n}\right)^{2}+\sum_{k=1}^{2}\left(\bar{u}_{j+1}^{\mathrm{D} 3 \mathrm{P} 1,(k)}-\bar{u}_{j}^{\mathrm{D} 3 \mathrm{P} 1,(k)}\right)^{2}+\left(\bar{u}_{j+1}^{\mathrm{D} 3 \mathrm{P} 1, n+1}-\bar{u}_{j}^{\mathrm{D} 3 \mathrm{P} 1, n+1}\right)^{2}, \\
& I_{j}^{x,-}=\left(\bar{u}_{j-1}^{\mathrm{D} 3 \mathrm{P} 1, n}-\bar{u}_{j}^{\mathrm{D} 3 \mathrm{P} 1, n}\right)^{2}+\sum_{k=1}^{2}\left(\bar{u}_{j-1}^{\mathrm{D} 3 \mathrm{P} 1,(k)}-\bar{u}_{j}^{\mathrm{D} 3 \mathrm{P} 1,(k)}\right)^{2}+\left(\bar{u}_{j-1}^{\mathrm{D} 3 \mathrm{P} 1, n+1}-\bar{u}_{j}^{\mathrm{D} 3 \mathrm{P} 1, n+1}\right)^{2}
\end{aligned}
$$

with $\bar{u}_{j}^{\mathrm{D} 3 \mathrm{P} 1,(k)}$ being the approximation at the $k$-th stage.

Finally, we point out that the coefficients $C_{L}$ and $C_{H}$ must be carefully chosen. In fact, since the time-limited solution (22) blends a first-order accurate solution with a third-order accurate one, according to [38] we must choose $C_{L}=\Delta t^{2}$ in order to obtain a third-order time limited solution. 


\subsubsection{Conservative Correction}

The two solutions $u^{\mathrm{IE}}$ and $u^{\mathrm{D} 3 \mathrm{P} 1}$ are obtained with conservative schemes, and thus conserve mass. However, the blending (23) itself is not conservative, because it occurs at the cell level, instead of at interfaces.

It is possible therefore that at the $j+1 / 2$ interface a mass loss (or gain) is observed. More precisely, the low order predictor can be written as

$$
u_{j}^{\mathrm{IE}, n+1}=\bar{u}_{j}^{n}-\frac{\Delta t}{h}\left(\mathcal{F}_{j+1 / 2}^{\mathrm{IE}}-\mathcal{F}_{j-1 / 2}^{\mathrm{IE}}\right),
$$

while the high order corrector is

$$
u_{j}^{\mathrm{D} 3 \mathrm{P} 1, n+1}=\bar{u}_{j}^{n}-\frac{\Delta t}{h}\left(\mathcal{F}_{j+1 / 2}^{\mathrm{D} 3 \mathrm{P} 1}-\mathcal{F}_{j-1 / 2}^{\mathrm{D} 3 \mathrm{P} 1}\right) .
$$

The blended solution therefore is

$$
u_{j}^{B, n+1}=\bar{u}_{j}^{n}-\frac{\Delta t}{h}\left[\frac{\omega_{j}^{H, n}}{C_{H}} \Delta \mathcal{F}_{j}^{\mathrm{D} 3 \mathrm{P} 1}+\left(\omega_{j}^{L}-\frac{C_{L}}{C_{H}} \omega_{j}^{H}\right) \Delta \mathcal{F}_{j}^{\mathrm{IE}}\right] .
$$

Since the blending is cell-centered, while the fluxes are based on the interfaces, we expect that through the $j+1 / 2$ interface there will be a mass loss (or gain) given by

$$
\mu_{j+1 / 2}^{n+1}=\frac{\Delta t}{C_{H} h}\left(\left(\omega_{j}^{H, n}-\omega_{j+1}^{H, n}\right) \mathcal{F}_{j+1 / 2}^{\mathrm{D} 3 \mathrm{P} 1}+\left(C_{H}\left(\omega_{j}^{L, n}-\omega_{j+1}^{L, n}\right)-C_{L}\left(\omega_{j}^{H, n}-\omega_{j+1}^{H, n}\right)\right) \mathcal{F}_{j+1 / 2}^{\mathrm{IE}}\right) .
$$

Thus we redistribute the mass lost through the $j+1 / 2$ interface among the $j$-th and $(j+1)$ th cell, obtaining the limited in time, limited in space Quinpi3 solution, which will be called Q3P1 solution. The redistribution is done proportionally to the high order nonlinear weight, so that

$$
u_{j}^{\mathrm{Q} 3 \mathrm{P} 1, n+1}=u_{j}^{B, n+1}+\frac{\omega_{j}^{H}}{\omega_{j}^{H}+\omega_{j+1}^{H}} \mu_{j+1 / 2}+\frac{\omega_{j}^{H}}{\omega_{j}^{H}+\omega_{j-1}^{H}} \mu_{j-1 / 2} .
$$

This is the updated solution at time $t^{n+1}$. The resulting scheme is conservative with numerical flux

$$
\begin{aligned}
\mathcal{F}_{j+1 / 2}^{\mathrm{Q} 3 \mathrm{P} 1}= & \frac{1}{C_{H}} \frac{2 \omega_{j}^{H} \omega_{j+1}^{H}}{\omega_{j}^{H}+\omega_{j+1}^{H}} \mathcal{F}_{j+1 / 2}^{\mathrm{D} 3 \mathrm{P} 1} \\
& +\frac{1}{C_{H}}\left(C_{H} \frac{\omega_{j}^{L} \omega_{j+1}^{H}+\omega_{j}^{H} \omega_{j+1}^{L}}{\omega_{j}^{H}+\omega_{j+1}^{H}}-C_{L} \frac{2 \omega_{j}^{H} \omega_{j+1}^{H}}{\omega_{j}^{H}+\omega_{j+1}^{H}}\right) \mathcal{F}_{j+1 / 2}^{\mathrm{IE}} .
\end{aligned}
$$

Introducing the "reduced mass" of the high order weights

$$
\tilde{\omega}_{j+1 / 2}^{H}=\frac{2 \omega_{j}^{H} \omega_{j+1}^{H}}{\omega_{j}^{H}+\omega_{j+1}^{H}},
$$

we can rewrite the conservative flux as 


$$
\mathcal{F}_{j+1 / 2}^{\mathrm{Q} 3 \mathrm{P} 1}=\frac{\tilde{\omega}_{j+1 / 2}^{H}}{C_{H}}\left[\mathcal{F}_{j+1 / 2}^{\mathrm{D} 3 \mathrm{P} 1}+\left(\frac{C_{H}}{2}\left(\frac{\omega_{j}^{L}}{\omega_{j}^{H}}+\frac{\omega_{j+1}^{L}}{\omega_{j+1}^{H}}\right)-C_{L}\right) \mathcal{F}_{j+1 / 2}^{\mathrm{IE}}\right] .
$$

From the formula, it is apparent that the high order flux of the interface contributes significantly to the time-limited flux only when the cells on both sides are detected as smooth. Moreover, it is also clear that $C_{L}$ must be infinitesimal.

In [5] an analogous conservative correction is employed to ensure the conservation property at interfaces between grid patches in an adaptive mesh refinement (AMR) algorithm. Other approaches are possible, in particular we refer to [30] which avoids a cell-centered blending by means of flux-based Runge-Kutta. Similar techniques are used in [3].

\section{Numerical Simulations}

The purpose of the tests appearing in this section is to study the accuracy of the Quinpi scheme proposed in this work, and to verify their smaller dissipation compared to the first-order predictor IE and their improved non-oscillatory properties compared to the non-limited in time corrector D3P1. Thus we will consider the standard tests which are commonly used in the literature on high order methods for conservation laws: linear advection of non-smooth waves, shock formation and interaction in Burgers' equation and the Buckley-Leverett non-convex equation. Furthermore, on one of the tests with singularities, see Fig. 7, we also demonstrate the need of the conservative correction discussed in the previous section.

As mentioned in the description of the scheme, when solving nonlinear conservation laws the solution of nonlinear systems is required both for the prediction and the correction steps. To this end, we employ Newton's method. The initial guess to start the iteration leading to $\bar{u}^{\mathrm{IE},(k)}$ is chosen as $\bar{u}^{\mathrm{IE},(k-1)}, k=2,3$. For the first stage, $k=1$, we use the high order solution emerging from the previous time step. The initial guess to compute the stages $\bar{u}^{(k)}$ of the corrector is the corresponding values $\bar{u}^{\mathrm{IE},(k)}$ of the predictor, for each $k=1,2,3$. The stopping criteria are based on the relative error between two successive approximations and on the norm of the residual. We use a given tolerance $\Delta t^{3}$, according to the global error of the scheme.

\subsection{Convergence Test}

We test the numerical convergence rate of the third-order Quinpi introduced in Sect. 3 on the nonlinear Burgers' equation

$$
u_{t}+\left(\frac{u}{2}\right)_{x}^{2}=0
$$

with the initial condition

$$
u_{0}(x)=0.5-0.25 \sin (\pi x)
$$


on $\Omega=[0,2]$ with the periodic domain, and up to the final time $t=1$, i.e., before the shock appears. The numerical errors, in both $L^{1}$ and $L^{\infty}$ norms, and convergence rates are showed in Table 1 for different CFL numbers.

In the nonlinear blending in time between the low-order solution, i.e., the composite implicit Euler (IE), and the high-order solution, i.e., D3P1, we use $C_{L}=\Delta t^{2}$ and $C_{H}=1-\Delta t^{2}$ as linear weights. We observe third-order convergence in both norms. In particular, we point out that with large CFL numbers our method reaches smaller errors and faster convergence with respect to the results in [3] for the same grid.

On the same smooth problem, we also test the convergence rate of the low-order predictor IE and the high-order corrector D3P1, separately. The convergence tests are shown in Fig. 1. Clearly, with IE we observe first-order accuracy. Instead, D3P1 achieves the optimal thirdorder accuracy, as expected.

\subsection{Linear Transport}

We consider the linear scalar conservation law

$$
u_{t}+u_{x}=0
$$

on the periodic domain in space $\Omega=[-1,1]$, and evolve the initial profile $u_{0}(x)$ for one period, i.e., up to the final time $t=2$. As the initial condition we consider the non-smooth profiles

$$
\begin{gathered}
u_{0}(x)=\sin (\pi x)+\left\{\begin{array}{l}
3,-0.4 \leqslant x \leqslant 0.4, \\
0, \text { otherwise }
\end{array}\right. \\
u_{0}(x)=\left\{\begin{array}{l}
1,-0.25 \leqslant x \leqslant 0.25, \\
0, \text { otherwise }
\end{array}\right.
\end{gathered}
$$

\begin{tabular}{|c|c|c|c|c|}
\hline$N$ & $L^{1}$ error & Rate & $L^{\infty}$ error & Rate \\
\hline \multicolumn{5}{|c|}{ (a) $\Delta t=h$} \\
\hline 640 & $2.24 \times 10^{-5}$ & 2.79 & $3.57 \times 10^{-4}$ & 2.62 \\
\hline 1280 & $2.91 \times 10^{-6}$ & 2.95 & $4.73 \times 10^{-5}$ & 2.91 \\
\hline 2560 & $3.66 \times 10^{-7}$ & 2.99 & $5.99 \times 10^{-6}$ & 2.98 \\
\hline \multicolumn{5}{|c|}{ (b) $\Delta t=10 h$} \\
\hline 320 & $3.59 \times 10^{-4}$ & 2.36 & $4.57 \times 10^{-3}$ & 1.76 \\
\hline 640 & $5.77 \times 10^{-5}$ & 2.64 & $9.65 \times 10^{-4}$ & 2.24 \\
\hline 1280 & $7.99 \times 10^{-6}$ & 2.85 & $1.56 \times 10^{-4}$ & 2.62 \\
\hline 2560 & $1.03 \times 10^{-6}$ & 2.96 & $2.12 \times 10^{-5}$ & 2.88 \\
\hline 5120 & $1.29 \times 10^{-7}$ & 2.99 & $2.70 \times 10^{-6}$ & 2.98 \\
\hline \multicolumn{5}{|c|}{ (c) $\Delta t=50 \mathrm{~h}$} \\
\hline 1280 & $5.67 \times 10^{-4}$ & 2.04 & $6.60 \times 10^{-3}$ & 1.49 \\
\hline 2560 & $9.73 \times 10^{-5}$ & 2.54 & $1.54 \times 10^{-3}$ & 2.10 \\
\hline 5120 & $1.41 \times 10^{-5}$ & 2.79 & $2.68 \times 10^{-4}$ & 2.52 \\
\hline
\end{tabular}

Table 1 Orders of convergence of the Quinpi scheme Q3P1 


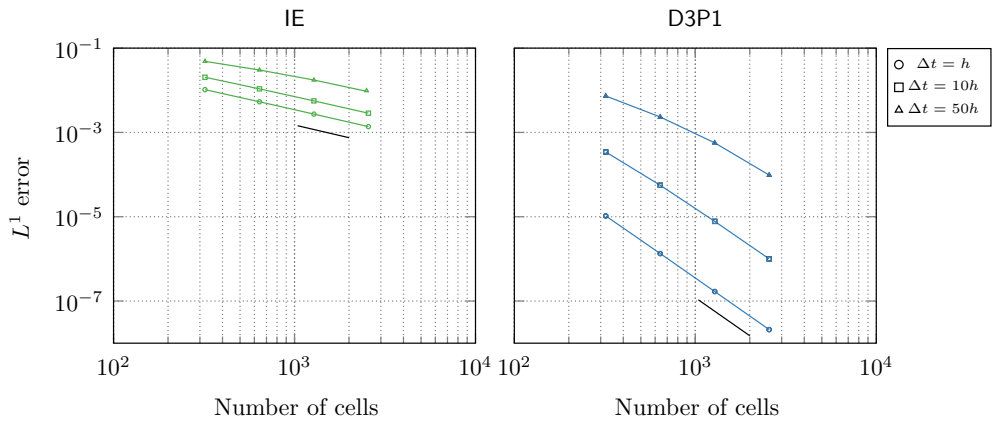

Fig. 1 Convergence test of the predictor scheme IE, left panel, and of the corrector scheme D3P1, right panel, for different CFL numbers

This problem is used to investigate the properties of a scheme to transport non-smooth data with minimal dissipation, dispersion and oscillation effects.

Figure 2 shows the numerical solutions of the linear transport problem with the initial profile (36a) computed with the predictor IE and with the corrector methods with no time limiting, i.e., D3P1 and with blending in time, i.e., Q3P1. We consider two different CFL numbers. All the solutions are computed on a grid of 400 cells. We observe that the low-order predictor is very diffusive, whereas the corrector D3P1 is oscillating across the discontinuities, in particular with CFL number 5. The corrector Q3P1 obtained after nonlinear blending of the IE and D3P1 solutions, is much less diffusive than IE and does not produce spurious oscillations, even with large CFL number.

In Fig. 3 we provide the numerical solutions of the linear transport problem with the initial double-step profile (36b) with a zoom on the top part of the non-smooth region of the solution. The simulations are performed with $\Delta t=5 h$. In the left panel, we compare the three methods on a grid of 400 cells. We observe that the novel method Q3P1 introduced in this work presents less oscillations than D3P1 close to discontinuities, and it is less dissipative than IE. The right panel shows the approximation provided by Q3P1 on different grids. As we expect, on finer grids the frequency of the oscillations increases, whereas the amplitude slightly decreases.
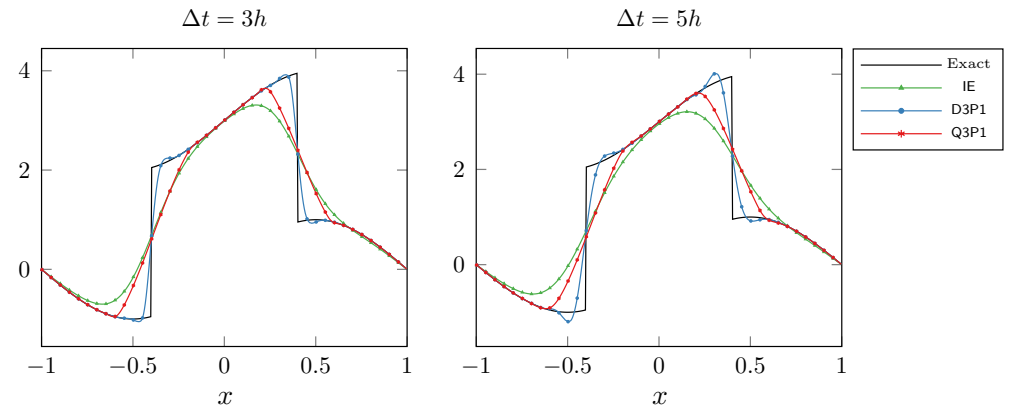

Fig. 2 Linear transport equation (35) with the initial condition (36a) on 400 cells at time $t=2$. The markers are used to distinguish the schemes, and are drawn one out of 10 cells 


\subsection{Burgers' Equation}

We investigate the behavior of the schemes on the nonlinear Burgers' equation (33) for different initial conditions.

\subsubsection{Smooth Profile: Shock Formation}

As in [3], we consider the smooth initial condition (34) on the periodic domain $\Omega=[0,2]$, and up to the final time $t=2$, i.e., after shock formation.

The results in Fig. 4 are obtained with 256 cells with two values of the CFL number. In both cases the Q3P1 scheme is slightly more diffusive than its non-blended version D3P1, and exhibits a much lower dissipation than the first-order predictor IE. For $\Delta t=3 h$, all schemes do not produce large oscillations near the discontinuity, and the solutions of Q3P1 and D3P1 are very close. We appreciate the difference when $\Delta t=5 h$. In fact, the Q3P1 scheme reduces the oscillations created by D3P1, while maintaining a very high resolution.

On this test, we show also the numerical approximation provided by the space-time non-limited scheme, cf. Fig. 5. The setup of the simulation is as in Fig. 4, i.e., we consider 256 cells with CFL numbers 3 and 5. Compared to Fig. 4, we observe the importance of the limiting technique to avoid the very large spurious oscillations appearing also at moderate CFL numbers.

\subsubsection{Non-smooth Profile}

We test the Burgers' equation on the discontinuous initial condition (36b), on the periodic domain $\Omega=[-1,1]$, and up to the final time $t=0.5$.

The numerical solutions are shown in Fig. 6 with 400 cells, with $\Delta t=3 h$ and $\Delta t=5 h$. The nonlinear Burgers' equation develops a rarefaction and a moving shock. Again, we observe the ability of the new implicit scheme Q3P1 of increasing the accuracy on smooth zones compared to IE, and, at the same time, reducing the spurious oscillations across the shock.
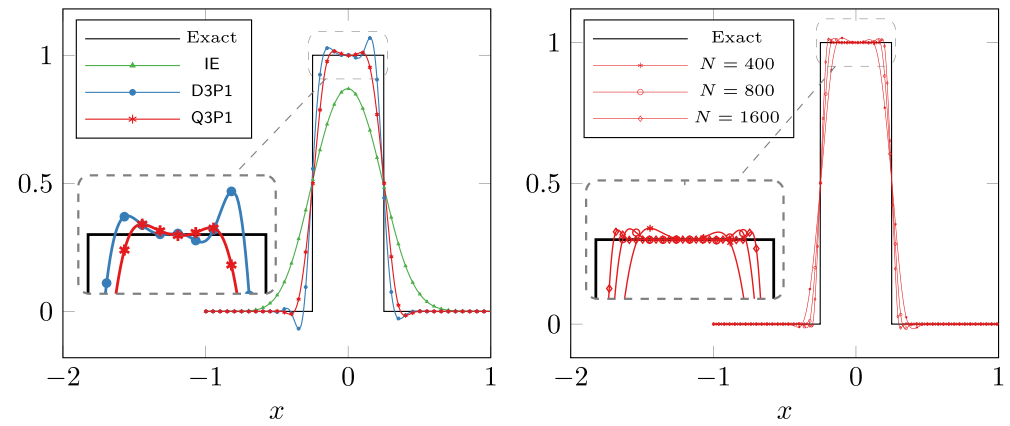

Fig. 3 Linear transport equation (35) with the initial condition (36b) with $\Delta t=5 h$ at time $t=2$. The solutions in the left panel are computed on 400 cells. The right panel shows the solutions obtained with the Q3P1 method on different grids. The markers are used to distinguish the schemes, and are drawn one out of 10 cells on the left and 15 cells on the right 

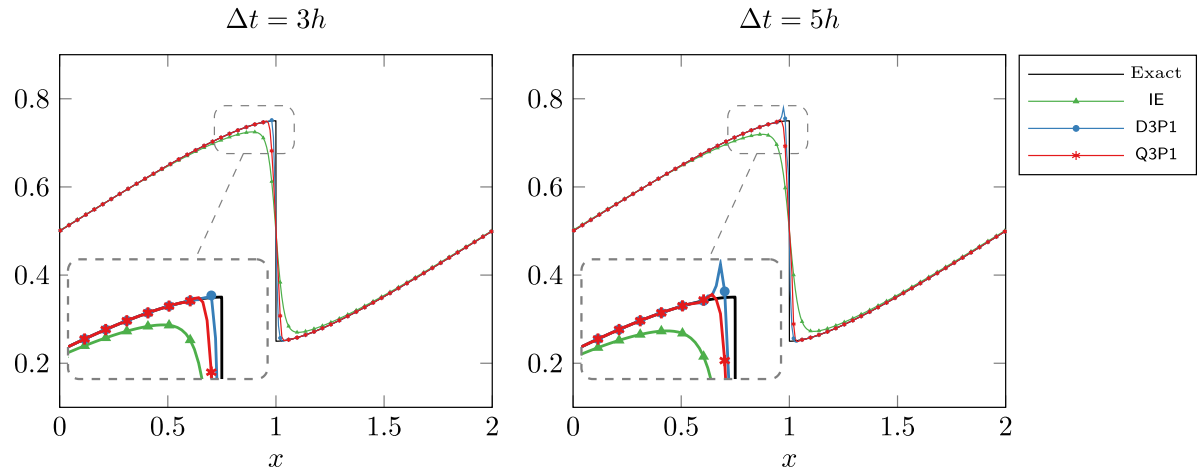

Fig. 4 Burgers' equation (33) with the initial condition (34) on $N=256$ cells at time $t=2$. The markers are used to distinguish the schemes, and are drawn each 5 cells
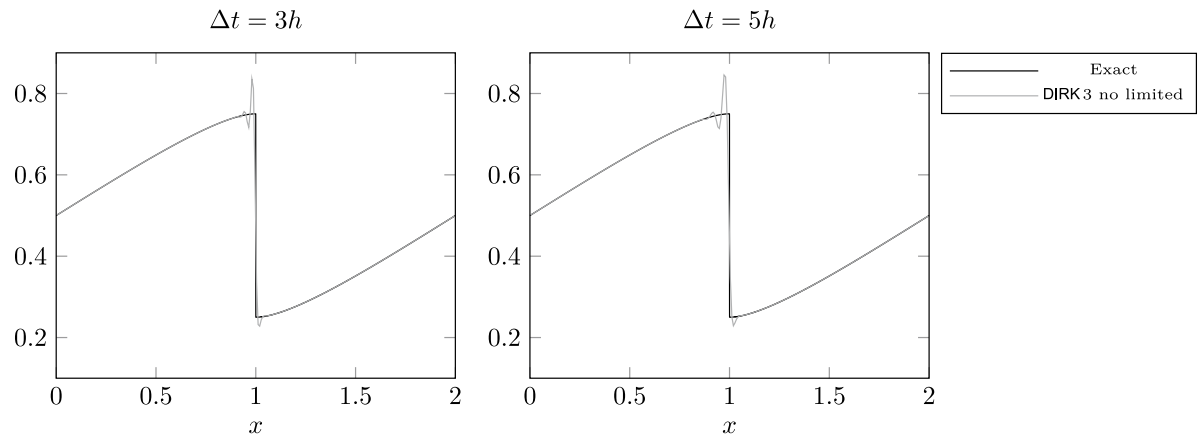

Fig. 5 Numerical solution of the Burgers' equation (33) with initial condition (34) on $N=256$ cells at time $t=2$ obtained with the space-time non-limited scheme

On this particular test, we provide a numerical evidence of the need and of the effectiveness of the conservative correction introduced after the nonlinear blending in time between the predictor scheme IE and the third-order corrector D3P1. In the right panel of Fig. 7 we show the solutions provided by the Quinpi scheme Q3P1 with and without the conservative correction, using 400 cells and $\Delta t=5 \mathrm{~h}$. Instead, in the left panel of Fig. 7 we show the behavior in time of the deviation of the mass of the numerical solution from the mass of the initial condition. We observe that, without correction, the Q3P1 scheme does not capture the correct shock location because of the mass lost. The conservative correction allows to predict the shock at the correct location and the mass is conserved at all time.

Finally, in Fig. 8 we show the numerical approximations obtained limiting the thirdorder scheme with an explicit first-order predictor as in [23], on a grid of 400 cells with $\Delta t=5 h$. The solutions are shown at two different time, and we observe that the use of an explicit predictor is not enough to prevent spurious oscillations in the high order scheme, at relatively high CFL numbers. At this CFL, the explicit predictor is already unstable, and thus the information it would provide on where to limit the solution is not reliable. 

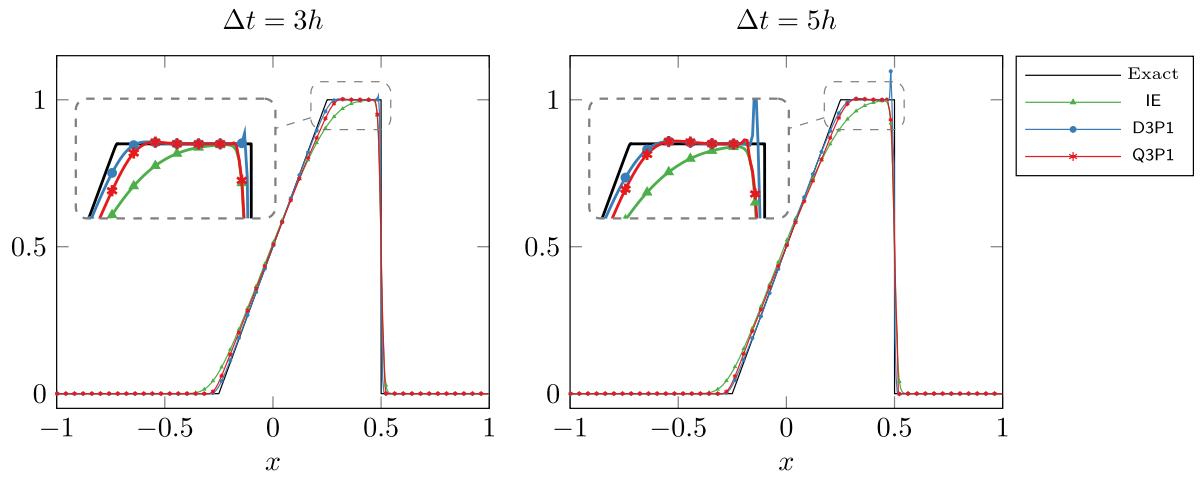

Fig. 6 Burgers' equation (33) with the initial condition (36b) on 400 cells at time $t=0.5$. The markers are used to distinguish the schemes, and are drawn every 8 cells
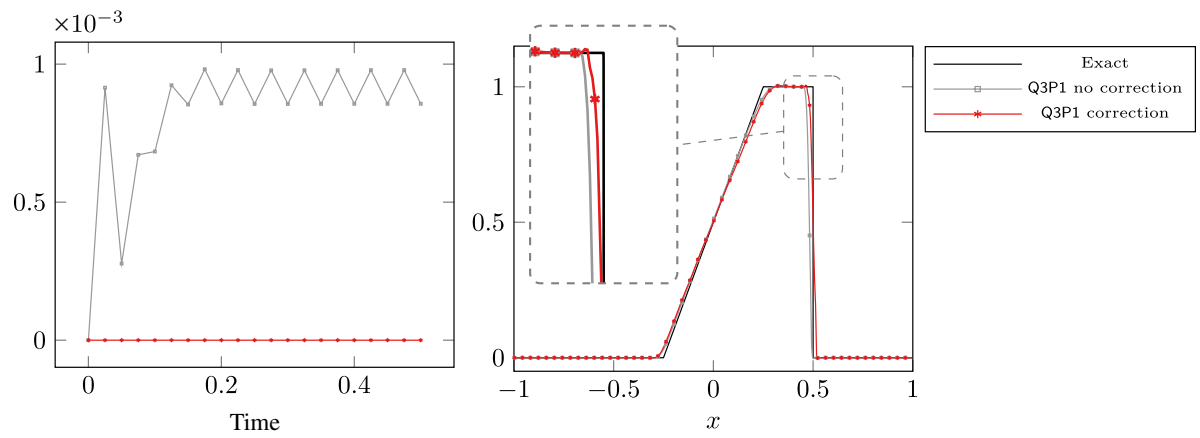

Fig. 7 Right plot: numerical solutions of Q3P1, with and without conservative correction, on the Burgers' equation (33) with the initial condition (36b) using 400 cells at time $t=5$ and with $\Delta t=5 h$. The markers are used to distinguish the schemes, and are drawn every 8 cells. Left plot: deviation in time of the total mass of the numerical solution from the exact total mass

\subsubsection{Shock Interaction}

We consider Burgers' equation with smooth initial condition

$$
u_{0}(x)=0.2-\sin (\pi x)+\sin (2 \pi x)
$$

on the periodic domain $\Omega=[-1,1]$, and with $\Delta t=5 h$. This test allows to compare the behavior of the schemes on both shock formation and shock interaction. In fact, the exact solution is characterized by the formation of two shocks which collide at a larger time, developing a single discontinuity.

In Fig. 9 we show the numerical solutions at three snapshots: at $t=\frac{1}{2 \pi}$, when the two shocks occur, at $t=0.6$, which is slightly before the interaction of the two shocks, and finally at $t=1$, shortly after the shock interaction. It is clear that Q3P1 does not produce spurious oscillations, and its profile has a higher resolution with respect to IE. 

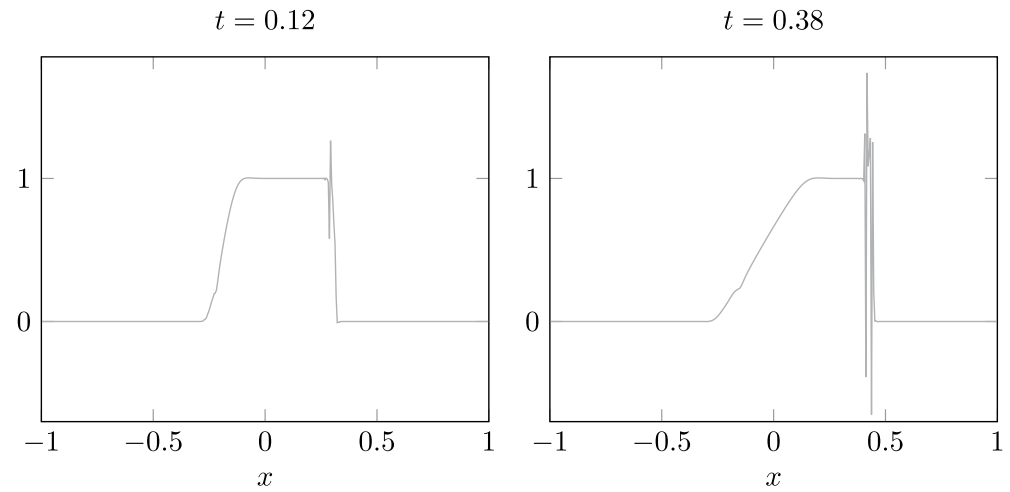

Fig. 8 Numerical solution of Burgers' equation (33) with the initial condition (36b) on 400 cells with $\Delta t=5 h$. The solution is limited according to the weights computed with an explicit first-order predictor, unlike the Quinpi approach which is characterized by implicit predictors

\subsection{Buckley-Leverett Equation}

We also show results on a non-convex problem, such as the Buckley-Leverett equation

$$
u_{t}+\left(\frac{u^{2}}{u^{2}+\frac{1}{3}(1-u)^{2}}\right)_{x}=0,
$$

which is characterized by a non-convex flux function. We consider the same setup as in [3]. Therefore, the initial profile is the step function

$$
u_{0}(x)=\left\{\begin{array}{l}
0.5, \quad-0.25 \leqslant x \leqslant 0.25, \\
0, \quad \text { otherwise }
\end{array}\right.
$$

on the periodic domain $\Omega=[0,1]$, and up to the final time $t=0.085$.

In Fig. 10 we show the results on a grid of 100 cells with $\Delta t=1.1 h$ and $\Delta t=4.4 h$. For this particular example, we set $\epsilon_{t}=\Delta t^{3}$ in the nonlinear blending in time. The results produced by the three schemes are very similar when the CFL is small. With large CFL, instead, we observe that Q3P1 improves D3P1 avoiding the spurious overshoots. However,
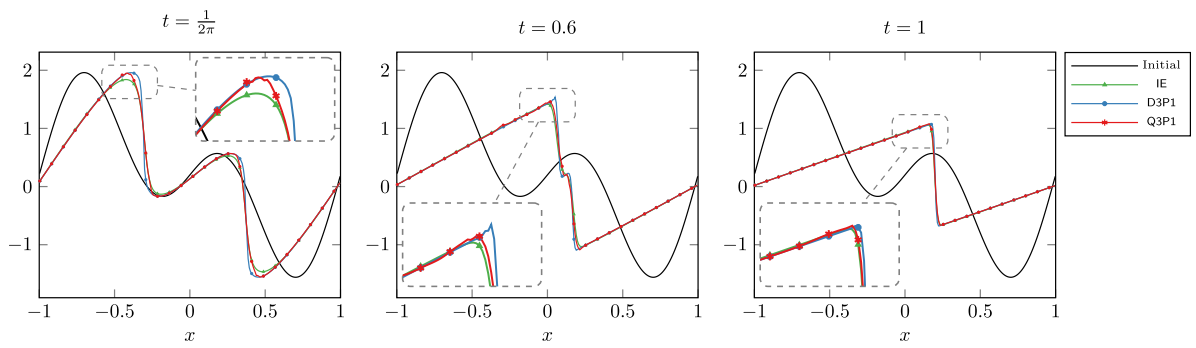

Fig. 9 Burgers' equation (33) with the initial condition (37) on 256 cells with $\Delta t=5 h$ at three different time. The markers are used to distinguish the schemes, and are drawn each 10 cells 
we note that on this particular test the Q3P1 and IE solutions almost coincide, in fact, the IE profile is hidden by Q3P1. Here the time limiting is dominating, probably because of the structure of the initial data.

\subsection{Computational Complexity}

The implicit method proposed in this paper is clearly more expensive compared to a corresponding explicit scheme, for a fixed number of time steps. But the benefit of an implicit scheme is to permit to use a larger CFL number, and thus a smaller number of time steps. This may result in more efficient computations for stiff problems.

When integrating a system of hyperbolic equations with a numerical scheme, one chooses the time step as

$$
\Delta t=\min \left(\Delta t_{\mathrm{acc}}, \Delta t_{\mathrm{stab}}\right),
$$

where $\Delta t_{\text {acc }}$ is fixed by accuracy constraints, and $\Delta t_{\text {stab }}$ is due to the CFL stability condition with

$$
\Delta t_{\text {stab }} \leqslant c \frac{h}{\left\|f^{\prime}(u)\right\|_{S}}
$$

where $\left\|f^{\prime}(u)\right\|_{S}$ is the spectral norm of the Jacobian of the flux. When $\left\|f^{\prime}(u)\right\|_{S} \gg 1$, the stability constraint is more demanding than accuracy, and implicit schemes may become convenient.

On scalar conservation laws, $\Delta t_{\text {acc }}$ and $\Delta t_{\text {stab }}$ have the same size, because there is only one propagation speed. Implicit integrators become of interest when there are fast waves one is not interested in, which however determine $\Delta t_{\text {stab }}$, while the phenomena one would like to resolve accurately are linked to slow waves, which then determine $\Delta t_{\text {acc }}$. In this case one is willing to tolerate a deterioration of the error on the fast waves, preserving accuracy on the slow waves. This is the case for instance for many solvers for low Mach or low Froude flows.

In a scalar problem, this improvement is not apparent. However, we can estimate the computational cost for a single time step of the explicit third-order SSP Runge-Kutta

$\Delta t=1.1 h$

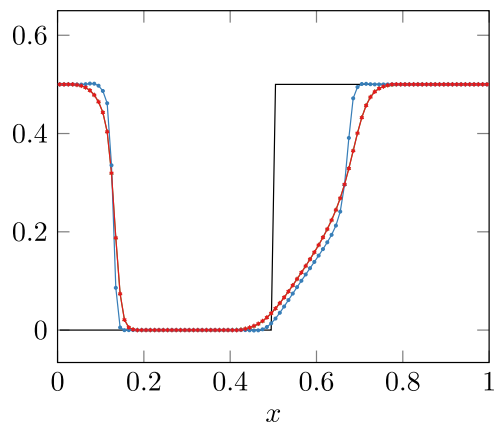

$\Delta t=4.4 h$

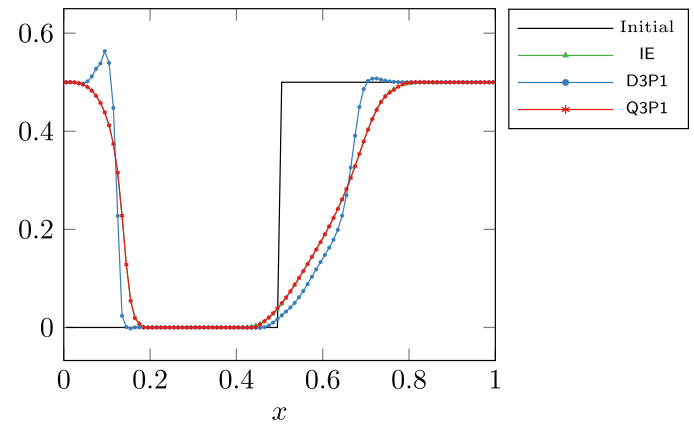

Fig. 10 Buckley-Leverett equation (38) with the initial condition (39) on 100 cells at time $t=0.085$ 
scheme and of the Q3P1 scheme. The ratio between these two costs will tell as at what CFL the implicit scheme becomes competitive.

In Table 2 we report the results obtained on the linear equation on $[-1,1]$ and on Burgers' equation on $[0,2]$ with the initial condition (37). The results are obtained on a quadcore Intel Core i7-6600U with clock speed $2.60 \mathrm{GHz}$.

As expected, a step of the implicit scheme is more expensive than a step of the explicit one. But several things can be noted. First of all, we expect that the implicit scheme should be faster on linear problems. In this case, in fact, no Newton iteration is needed, thanks to the linearity of the predictor. This is confirmed by our data. Moreover, both the explicit and the implicit schemes should have a computational cost scaling as $N$, and this is true for Q3P1. Apparently the computational cost scales more favorably for the explicit scheme, but this can be due to more efficient memory operations whose analysis goes beyond the purpose of this work.

The complexity of the implicit scheme increases on a nonlinear problem. In fact, the Q3P1 scheme requires approximately six Newton's iterations each time step. However, the number of iterations remains bounded, and, most important, it does not depend on $N$. The computational cost increases slightly for the non-smooth problem: in this case, in fact, the initial guess of the first Newton's method is given by the solution obtained at the previous time step, which may not be accurate in the presence of shocks.

In any case, it is clear that the Q3P1 scheme becomes faster for CFL numbers larger than 5 or 6 in the nonlinear case, and even before in the linear case. Since stiff problems, such as low Mach, can have CFLs of order hundreads and even more, it is to be expected that the implicit scheme can be convenient in many applications.

In Fig. 11 we show the total number of iterations required for Newton's method in each time step as a function of time in solving Burgers' equation with the smooth (37) and non-smooth (36b) initial profiles up to $t=0.5$ and with three different Courant numbers. We recall that a nonlinear system must be solved at each predictor step, and at each Runge Kutta stage. Thus a total of 6 nonlinear systems of algebraic equations must be solved at each time step of a third-order scheme.

Table 2 CPU time for each step of the SSP-RK3 explicit scheme and of the Q3P1 implicit scheme on linear and nonlinear problems

\begin{tabular}{lccc}
\hline Cells $N$ & SSP-RK3 & Q3P1 & Ratio $r_{N}$ \\
\hline $\begin{array}{l}\text { (a) Linear problem } \\
200\end{array}$ & & \\
400 & 0.0021 & 0.0062 & 2.95 \\
800 & 0.0033 & 0.0093 & 2.82 \\
1600 & 0.0061 & 0.0155 & 2.54 \\
(b) Nonlinear problem before shock formation & 3.08 \\
200 & 0.0095 & 0.0293 & \\
400 & 0.0023 & 0.0103 & 4.48 \\
800 & 0.0032 & 0.0148 & 4.62 \\
1600 & 0.0066 & 0.0229 & 3.47 \\
(c) Nonlinear problem after shock formation & 3.97 \\
200 & 0.0091 & 0.0361 & \\
400 & 0.0023 & 0.0107 & 4.65 \\
800 & 0.0033 & 0.0158 & 4.79 \\
1600 & 0.0068 & 0.0255 & 3.75 \\
\hline
\end{tabular}


The top panels of Fig. 11 are obtained with 400 cells, whereas the bottom panels are with 800 cells.

For the smooth initial condition, we observe that the number of iterations is not larger than 2 for each Newton's application as long as the solution remains smooth. Instead, the number of iterations slightly increases when the solution becomes discontinuous. In fact, each Newton's method is converging with maximum 3 iterations. For the doublestep initial condition, the number of iterations is larger, but no more than 3 iterations are needed on average for each Newton's solution.

But the most important point is that the number of iterations of Newton's method is independent of the number of cells and of the CFL. Thus the implicit method becomes more and more convenient as the stiffness of the method increases.

\section{Conclusions}

In this work, we have proposed a new approach to the integration of hyperbolic conservation laws with high order implicit schemes. The main characteristic of this framework is to use low order implicit predictors with a double purpose. First, the predictor is used to determine the nonlinear weights in a CWENO or WENO high order space reconstruction. In this fashion, one greatly simplifies the differentiation of the WENO weights when computing the Jacobian of the numerical fluxes. Second, the predictor is used as
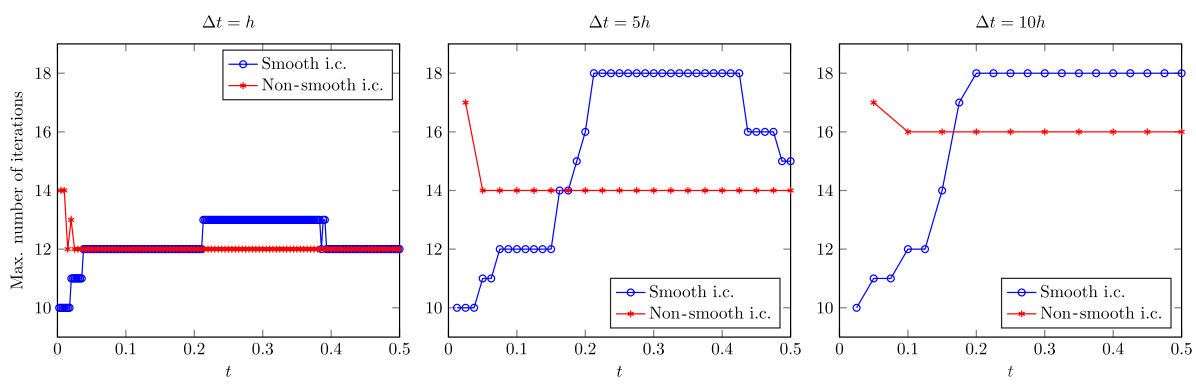

(a) 400 cells
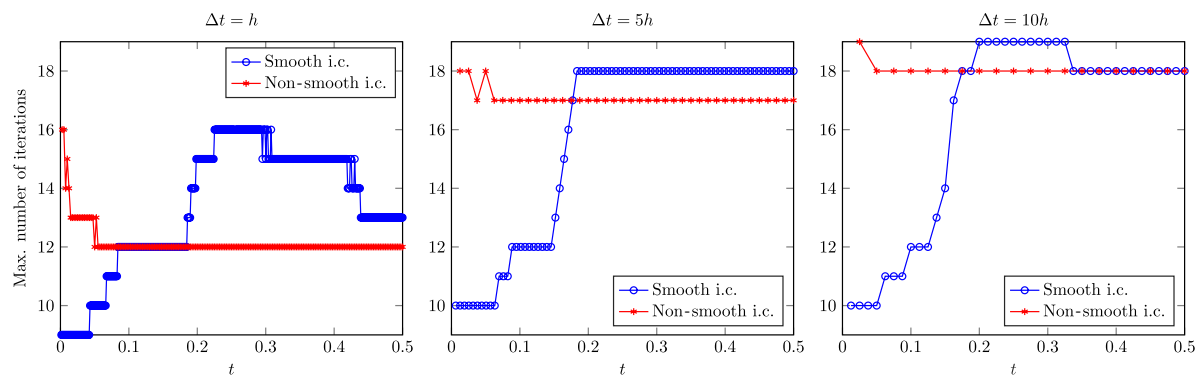

(b) 800 cells

Fig. 11 Total number of iterations in each time step required for the convergence of Newton's method when solving Burgers' equation up to $t=0.5$, with the initial condition (37) (blue lines) and (36b) (red lines). Three different values of the Courant number are considered 
low order approximation of the solution and is blended with the high order solution to achieve limiting also in time.

The resulting scheme is linear with respect to the solution at the new time level on linear equations, unlike most, if not all, high order implicit schemes available. The nonlinearity of the scheme is linked only to the nonlinearity of the flux. This does not mean that the coefficients appearing in the scheme are constant. It means that the nonlinearities in the limiting in space and time of the scheme involve only the predictor, which is already known when the high order solution is evolved in time.

We expect the new scheme to have applications in many stiff problems, as low Mach gas dynamics or kinetic problems. In these cases, one is not interested in the accuracy of the fast waves which determine the stiffness of the system, but would like to use a time step tailored to preserve the accuracy of the slow material waves.

Future work will involve the application of the Quinpi approach to stiff gas dynamics, the exploration of this new framework with BDF time integration, and extensions to a higher order.

Acknowledgements This work was partly supported by MIUR (Ministry of University and Research) PRIN2017 project number 2017KKJP4X, and Progetto di Ateneo Sapienza, number RM120172B41DBF3A.

Funding Open access funding provided by Università degli Studi di Roma La Sapienza within the CRUICARE Agreement.

\section{Compliance with Ethical Standards}

Conflict of Interest The authors declare that they have no conflict of interest.

Open Access This article is licensed under a Creative Commons Attribution 4.0 International License, which permits use, sharing, adaptation, distribution and reproduction in any medium or format, as long as you give appropriate credit to the original author(s) and the source, provide a link to the Creative Commons licence, and indicate if changes were made. The images or other third party material in this article are included in the article's Creative Commons licence, unless indicated otherwise in a credit line to the material. If material is not included in the article's Creative Commons licence and your intended use is not permitted by statutory regulation or exceeds the permitted use, you will need to obtain permission directly from the copyright holder. To view a copy of this licence, visit http://creativecommons.org/licenses/by/4.0/.

\section{References}

1. Abbate, E., Iollo, A., Puppo, G.: An all-speed relaxation scheme for gases and compressible materials. J. Comput. Phys. 351, 1-24 (2017)

2. Alexander, R.: Diagonally implicit Runge-Kutta methods for stiff O.D.E.'s. SIAM J. Numer. Anal. 14(6), 1006-1021 (1977)

3. Arbogast, T., Huang, C., Zhao, X., King, D.N.: A third order, implicit, finite volume, adaptive Runge-Kutta WENO scheme for advection-diffusion equations. Comput. Methods Appl. Mech. Eng. (2020). https://doi.org/10.1016/j.cma.2020.113155

4. Balsara, D.S., Garain, S., Shu, C.-W.: An efficient class of WENO schemes with adaptive order. J. Comput. Phys. 326, 780-804 (2016)

5. Berger, M.J., LeVeque, R.J.: Adaptive mesh refinement using wave-propagation algorithms for hyperbolic systems. SIAM J. Numer. Anal. 35(6), 2298-2316 (1998). https://doi.org/10.1137/ S0036142997315974

6. Boscarino, S., Cho, S., Russo, G., Yun, S.: High order conservative semi-Lagrangian scheme for the BGK model of the Boltzmann equation. Commun. Comput. Phys. 29, 1-56 (2021) 
7. Boscarino, S., Russo, G., Scandurra, L.: All Mach number second order semi-implicit scheme for the Euler equations of gas dynamics. J. Sci. Comput. 77(2), 850-884 (2018)

8. Carlini, E., Ferretti, R., Russo, G.: A weighted essentially nonoscillatory, large time-step scheme for Hamilton-Jacobi equations. SIAM J. Sci. Comput. 27, 1071-1091 (2005)

9. Castro-Dìaz, M.J., Semplice, M.: Third- and fourth-order well-balanced schemes for the shallow water equations based on the CWENO reconstruction. Int. J. Numer. Meth. Fluid 89(8), 304-325 (2019). https://doi.org/10.1002/fld.4700

10. Cravero, I., Puppo, G., Semplice, M., Visconti, G.: CWENO: uniformly accurate reconstructions for balance laws. Math. Comput. 87(312), 1689-1719 (2018). https://doi.org/10.1090/mcom/3273

11. Cravero, I., Semplice, M.: On the accuracy of WENO and CWENO reconstructions of third order on nonuniform meshes. J. Sci. Comput. 67, 1219-1246 (2016). https://doi.org/10.1007/ s10915-015-0123-3

12. Cravero, I., Semplice, M., Visconti, G.: Optimal definition of the nonlinear weights in multidimensional central WENOZ reconstructions. SIAM J. Numer. Anal. 57(5), 2328-2358 (2019). https://doi. org/10.1007/s10915-015-0123-3

13. Degond, P., Tang, M.: All speed scheme for the low Mach number limit of the isentropic Euler equations. Commun. Comput. Phys. 10(1), 1-31 (2011)

14. Dellacherie, S.: Analysis of Godunov type schemes applied to the compressible Euler system at low Mach number. J. Comput. Phys. 229(4), 978-1016 (2010)

15. Dimarco, G., Loubère, R., Vignal, M.H.: Study of a new asymptotic preserving scheme for the Euler system in the low Mach number limit. SIAM J. Sci. Comput. 39(5), A2099-A2128 (2017)

16. Dimarco, G., Loubère, R., Michel-Dansac, V., Vignal, M.H.: Second-order implicit-explicit total variation diminishing schemes for the Euler system in the low Mach regime. J. Comput. Phys. 372, 178-201 (2018)

17. Dimarco, G., Pareschi, L.: Numerical methods for kinetic equations. Acta Numer. 23, 369-520 (2014). https://doi.org/10.1017/S0962492914000063

18. Dumbser, M., Balsara, D.S., Toro, E.F., Munz, C.D.: A unified framework for the construction of onestep finite volume and discontinuous Galerkin schemes on unstructured meshes. J. Comput. Phys. 227, 8209-8253 (2008). https://doi.org/10.1016/j.jcp.2008.05.025

19. Dumbser, M., Boscheri, W., Semplice, M., Russo, G.: Central weighted ENO schemes for hyperbolic conservation laws on fixed and moving unstructered meshes. SIAM J. Sci. Comput. 39(6), A2564A2591 (2017). https://doi.org/10.1137/17M1111036

20. Duraisamy, K., Baeder, D.: Implicit scheme for hyperbolic conservation laws using non-oscillatory reconstruction in space and time. SIAM J. Sci. Comput. 29, 2607-2620 (2007)

21. Duraisamy, K., Baeder, D., Liu, J.: Concepts and application of time-limiters to high resolution schemes. J. Sci. Comput. 19, 139-162 (2003)

22. Forth, S.A.: A second order accurate space-time limited BDF scheme for the linear advection equation. In: Godunov Methods, pp. 335-342. Springer, Berlin (2001)

23. Gottlieb, S., Mullen, J.S., Ruuth, S.J.: A fifth order flux implicit WENO method. J. Sci. Comput. 27, 271-287 (2006). https://doi.org/10.1007/s10915-005-9034-z

24. Gottlieb, S., Shu, C.-W., Tadmor, E.: Strong stability preserving high-order time discretization methods. SIAM Rev. 43, 73-85 (2001)

25. Groppi, M., Russo, G., Stracquadanio, G.: High order semi-Lagrangian methods for the BGK equation. Commun. Math. Sci. 14, 389-414 (2016)

26. Harten, A.: High resolution schemes for hyperbolic conservation laws. J. Comput. Phys. 49(3), 357393 (1983). https://doi.org/10.1016/0021-9991(83)90136-5

27. Harten, A.: On a class of high resolution total-variation-stable finite-difference schemes. SIAM J. Numer. Anal. 21, 1-23 (1984)

28. Jiang, G.S., Shu, C.-W.: Efficient implementation of weighted ENO schemes. J. Comput. Phys. 126, 202-228 (1996)

29. Jin, S., Xin, Z.: The relaxation schemes for systems of conservation laws in arbitrary space dimensions. Commun. Pure Appl. Math. 48(3), 235-276 (1995)

30. Ketcheson, D.I., MacDonald, C.B., Ruuth, S.J.: Spatially partitioned embedded Runge-Kutta methods. SIAM J. Numer. Anal. 51(5), 2887-2910 (2013). https://doi.org/10.1137/130906258

31. Kolb, O.: On the full and global accuracy of a compact third order WENO scheme. SIAM J. Numer. Anal. 52(5), 2335-2355 (2014). https://doi.org/10.1137/130947568

32. LeVeque, R.: Finite volume methods for hyperbolic problems. In: Cambridge Texts in Applied Mathematics. Cambridge University Press, Cambridge (2004)

33. Lemou, M., Mieussens, L.: A new asymptotic preserving scheme based on micro-macro formulation for linear kinetic equations in the diffusion limit. SIAM J. Sci. Comput. 31, 334-368 (2008) 
34. Levy, D., Puppo, G., Russo, G.: Compact central WENO schemes for multidimensional conservation laws. SIAM J. Sci. Comput. 22(2), 656-672 (2000). https://doi.org/10.1137/S1064827599359461

35. Nørsett, S.P., Wanner, G.: Perturbed collocation and Runge-Kutta methods. Numer. Math. 38, 193-208 (1981). https://doi.org/10.1007/BF01397089

36. Pieraccini, S., Puppo, G.: Microscopically implicit-macroscopically explicit schemes for the BGK equation. J. Comput. Phys. 231, 299-327 (2012)

37. Semplice, M., Coco, A., Russo, G.: Adaptive mesh refinement for hyperbolic systems based on thirdorder compact WENO reconstruction. J. Sci. Comput. 66, 692-724 (2016). https://doi.org/10.1007/ s10915-015-0038-z

38. Semplice, M., Visconti, G.: Efficient implementation of adaptive order reconstructions. J. Sci. Comput. 83, 6 (2020). https://doi.org/10.1007/s10915-020-01156-6

39. Shu, C.-W.: Essentially non-oscillatory and weighted essentially non-oscillatory schemes for hyperbolic conservation laws. In: Advanced Numerical Approximation of Nonlinear Hyperbolic Equations (Cetraro, 1997), Lecture Notes in Math., vol. 1697, pp. 325-432. Springer, Berlin (1998)

40. Sweby, P.: High resolution schemes using flux limiters for hyperbolic conservation laws. SIAM J. Numer. Anal. 21(5), 995-1011 (1984). https://doi.org/10.1137/0721062

41. Tavelli, M., Dumbser, M.: A pressure-based semi-implicit space-time discontinuous Galerkin method on staggered unstructured meshes for the solution of the compressible Navier-Stokes equations at all Mach numbers. J. Comput. Phys. 341, 341-376 (2017). https://doi.org/10.1016/j.jcp.2017.03.030

42. Zennaro, M.: Natural continuous extensions of Runge-Kutta methods. Math. Comput. 46, 119-133 (1986) 